\title{
Therapeutic Effect of Low-Energy Nitrogen Plasma Pulses on Tinea Pedis
}

Heesu Kim ${ }^{1}$

Hyun-Jo Kim²

Sung Bin Cho ${ }^{1,3}$

${ }^{1}$ Department of Dermatology and Cutaneous Biology Research Center, International St. Mary's Hospital, Catholic Kwandong University College of Medicine, Incheon, Korea

${ }^{2}$ CNP Skin Clinic, Cheonan, Korea

${ }^{3}$ Yonsei Seran Dermatology and Laser Clinic, Seoul, Korea
Received May 15, 2019

Accepted May 23, 2019

\section{Correspondence}

Sung Bin Cho

Department of Dermatology and Cutaneous

Biology Research Center, International St. Mary's Hospital, Catholic Kwandong University College of Medicine, 25 Simgok-ro, Seo-gu, Incheon 22711, Korea

Tel.: +82-32-290-3141

Fax: +82-32-290-3142

E-mail: drsbchođggmail.com

(c) Korean Society for Laser Medicine and Surgery

(c) This is an open access article distributed under the terms of the Creative Commons Attribution NonCommercial License (http://creativecommons.org/ licenses/by-nc/4.0) which permits unrestricted noncommercial use, distribution, and reproduction in any medium, provided the original work is properly cited.
Superficial fungal infections with dermatophytes, nondermatophyte molds, or yeasts are treated primarily with topical and/or systemic antifungal agents. Additional or alternative treatment modalities, particularly energy-delivering modalities, however, are used widely to induce fungicidal effects via selective photothermal reactions. In addition to light- or laser-based devices, plasma therapy also has antifungal properties. This report describes a Korean male patient with mycologically confirmed tinea pedis that was treated effectively with two sessions of nitrogen plasma treatment at one-week intervals using a plasma delivering system. Nitrogen plasma was prepared by loading a 0.28- $\mathrm{ml}$ inert nitrogen gas/pulse that was activated by a microwave generator. The other treatment settings were a nozzle diameter of $5 \mathrm{~mm}$, pulse energy of $0.75 \mathrm{~J}$, pulse duration of $7 \mathrm{msec}$, and two passes. One week after the first session of nitrogen plasma treatment, the patient exhibited marked reductions in scale and inflammation. One month after the final treatment, no clinical features of recurrence were found, and successive potassium hydroxide testing revealed negative results.

\section{Key words}

Plasma; Nitrogen; Tinea pedis; Fungus 


\section{INTRODUCTION}

Superficial fungal infections of the skin are common and can be caused by dermatophytes, nondermatophyte molds, and yeasts. ${ }^{1,2}$ Treatment modalities for superficial fungal infections primarily rely on topical and/or systemic antifungal agents for eliminating fungal organisms and recovering the skin or nails as they grow. ${ }^{1,3}$ However, systemic antifungal agents pose risks of drug interactions and adverse events, including skin rash, headache, liver enzyme abnormalities, gastrointestinal discomfort, and taste disturbances. ${ }^{3}$ Thus, additional or alternative treatment modalities, particularly energy-delivering modalities, have been investigated.

Lasers have been approved by the US Food and Drug Administration for temporary clearing, but not curing, of onychomycosis. ${ }^{3}$ Nonetheless, near infrared diode lasers and 1,064-nm neodymium:yttrium-aluminum-garnet (Nd:YAG) lasers have been widely used to theoretically induce fungicidal effects via selective photothermal effects: fungal chromophores, which are thought to absorb laser energy, include melanin, chitin, and xanthomegnin. ${ }^{3-6}$

In addition to light- or laser-based devices, plasma therapy for treating toenail fungal infections has also been investigated. ${ }^{7.8}$ Plasma is generated by stripping electrons from atoms from inert gaseous sources and has been found to hold antifungal properties. ${ }^{3,9}$ Nonetheless, the clinical efficacy and safety of plasma energy on superficial fungal infections in the skin have not been fully elucidated. In this report, we describe a Korean male patient with mycologically confirmed tinea pedis that was effectively treated with pulsed delivery of nitrogen plasma.

\section{CASE REPORT}

A 40-year-old Korean male visited our clinic presenting with well-demarcated, erythematous to skin-colored, scaly patches on his right sole. The patient was diagnosed with mycologically confirmed tinea pedis by a potassium hydroxide test. He had been treated with oral itraconazole and topical econazole cream. However, the clinical outcomes thereof were unsatisfactory, and the lesions frequently recurred. He had never undergone treatment with laser- or light-assisted antifungal treatments, including 405- or 635-nm light-emitting diode modules and 1,064- or 1,444-nm Nd:YAG lasers, or argon and nitrogen plasma treatments. He had no pertinent history of use of systemic and topical antifungal agents. Moreover, compared to an initial visitation, the patient presented with more extensive scaly patches distributed wider across his right sole (Fig. 1A).

After obtaining written informed consent, the patient was treated with two sessions of nitrogen plasma treatment at one-week intervals using a plasma delivering system (Pladuo ${ }^{T M}$; Shenb Co., Ltd., Seoul, Korea) that generates plasma from an argon or nitrogen gas source using a $2.45-\mathrm{GHz}$ high-frequency microwave plasma generator. Nitrogen plasma was prepared by loading a $0.28-\mathrm{ml}$ inert nitrogen gas/pulse that was activated by a microwave generator. The other treatment settings included a nozzle diameter of $5 \mathrm{~mm}$, a pulse energy of $0.75 \mathrm{~J}$, and a pulse duration of $7 \mathrm{msec}$. After cleansing the
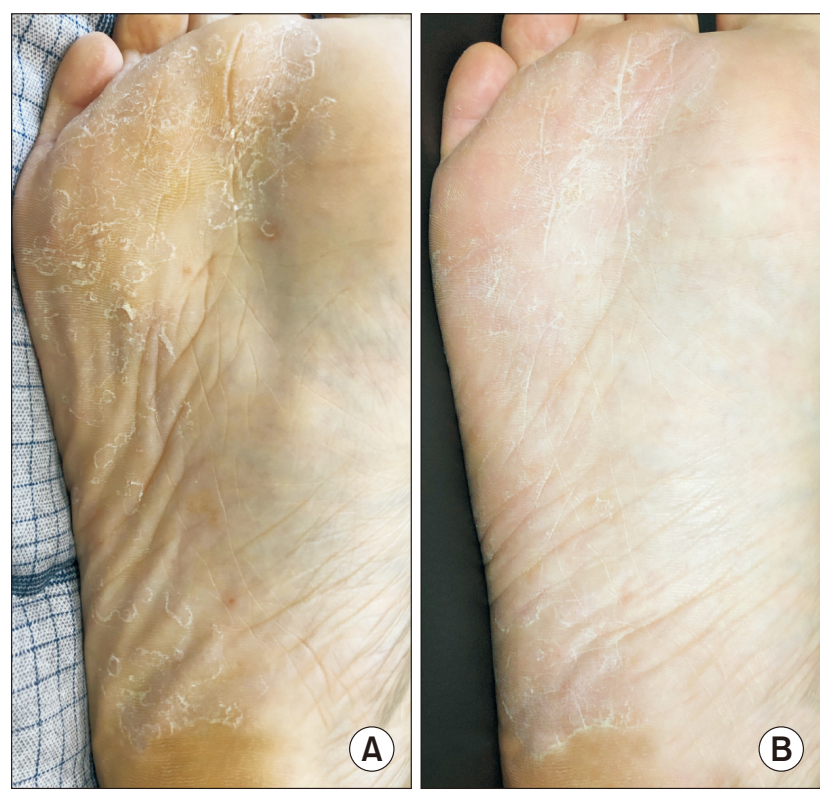

Fig. 1. Photographs of the right sole of a 40-year-old Korean male patient (A) at baseline, (B) one week after an initial session of nitrogen plasma treatment, and $(\mathrm{C})$ one week after a second session of nitrogen plasma treatment. Nitrogen plasma treatments were performed with a loading volume of 0.28-ml inert nitrogen gas/pulse, a pulse energy of $0.75 \mathrm{~J}$, a pulse duration of $7 \mathrm{msec}$, a nozzle diameter of 5 $\mathrm{mm}$, and a distance from the nozzle's tip to the skin of $10 \mathrm{~mm}$ over two passes. Photographs were taken under normal light exposure. 
patient's right foot with $70 \%$ ethanol, the entire sole was treated with two passes of nitrogen plasma pulse treatment at a distance from the nozzle's tip to the skin of 10 $\mathrm{mm}$ without pretreatment with topical anesthetic cream. Neither posttreatment cooling nor prophylactic prescription of systemic or topical corticosteroids, antibiotics, or antifungal agents was made.

One week after the first session of nitrogen plasma treatment, the patient exhibited marked reductions in scales and inflammation (Fig. 1B). Furthermore, subjective itching sensations had subsided within one day. One week after the second session of nitrogen plasma treatment, the lesions on the sole had further improved, and the patient was satisfied with the results (Fig. 1C). One month after the last treatment, no clinical features of recurrence were recorded, and successive potassium hydroxide testing revealed negative results. Pain during the treatment was unremarkable without the use of topical anesthetic cream, and the patient reported only a warm sensation during the delivery of nitrogen plasma pulses. Furthermore, no remarkable major side effects, including itching, crusts, blistering, oozing, burn, wound infection, prolonged edema or erythema, postinflammatory dyschromia, or scarring, were encountered.

\section{DISCUSSION}

Plasma is the fourth state of matter and consists of charged electrons, ions, nitric oxide, and hydroxyl radicals. ${ }^{3,7-9}$ Several inert gaseous sources, including ambient air, argon, helium, and nitrogen, have been used for generating plasma for various medical purposes by stripping electrons from atoms using pulses of high-frequency radiofrequency or microwave energy. ${ }^{3,9}$ Argon plasma has been used for promoting wound repair, inducing antibacterial, antiviral, and antifungal effects, and reducing pruritic skin reactions. ${ }^{10}$ Meanwhile, nitrogen plasma has been clinically used in the treatment of photodamaged skin and wrinkles by generating distinctive chromophoreindependent, energy-dependent zones of thermal damage and modification. . $^{\text {9 }}$

Plasma treatment in a previous in vitro study was found to significantly inhibit the growth of Trichophyton rubrum, which is the most common causative organism for onychomycosis. ${ }^{3,8}$ Additionally, $53.8 \%$ of the patients with onychomycosis of the toenails were clinically cured with three sessions of nonthermal plasma treatment at oneweek intervals. ${ }^{3,8}$ Nonetheless, the use of high-energy or thermal plasma for treating onychomycosis has been limited by the risk of intolerable pain during treatment and extensive tissue heating that causes irreversible damage to the nail matrix and bed. ${ }^{3}$

In the present report, we described a Korean male patient with mycologically confirmed tinea pedis that was effectively treated with pulsed delivery of low-energy nitrogen plasma. The patient's sole was treated with two passes of nitrogen plasma at a pulse energy of $0.75 \mathrm{~J}$ and a pulse duration of $7 \mathrm{msec}$. In a previous study, nitrogen plasma was found to induce a central zone of irreversible thermal damage that extended from the epidermis to the dermis depending on the energy settings per pulse. ${ }^{11} \mathrm{Ac}$ cordingly, we discerned that an irreversible thermal tissue reaction could be generated in the stratum corneum and upper part of the epidermis, which might contain fungal hyphae and spores.

In our patient, scales of tinea pedis in the skin became rougher over 3 to 5 days post treatment and spontaneously disappeared within 7 days. Meanwhile, itching sensations, which are associated with superficial fungal infection, seemed to improve more rapidly than the improvement in the skin scales. Based on the post-plasma clinical course, we deemed that the pulses of nitrogen plasma could have affected the uppermost epidermis, directly causing damage to fungal hyphae and pores. Moreover, the use of low-energy nitrogen plasma elicited no remarkable pain or heat sensation during the treatments and no downtime, compared to high-energy nitrogen plasma. Thus, nitrogen plasma treatment can be considered as an additive and safe treatment modality to conventional topical and systemic antifungal agents for treating tinea pedis infections.

In conclusion, we report a case of refractory tinea pedis that was effectively treated with pulsed delivery of low-energy nitrogen plasma. However, because well-designed studies comparing the efficacy and safety of low-energy nitrogen plasma treatment versus topical and systemic antifungal agents are lacking, nitrogen plasma should not be used as a first-line treatment for tinea pedis. Notwithstanding, our results suggest that nitrogen plasma is not only effective and safe for improving hyperkeratotic skin disorders, but also can directly damage fungal hyphae and spores. Further controlled studies are needed to investigate the efficacy and safety of nitrogen plasma treatment for superficial fungal infections of the skin.

\section{ACKNOWLEDGEMENTS}

We would like to thank Bora Kim (Shenb Co., Ltd., Seoul, Koreal and Min Choi (Shenb Co.) for their assistance with technical support. We would also like to thank 
Anthony Thomas Milliken, ELS at Editing Synthase (https:// editingsynthase.com) for his help with the editing of this manuscript.

\section{CONFLICTS OF INTEREST}

The authors declare no conflicts of interest.

\section{REFERENCES}

1. Foster KW, Ghannoum MA, Elewski BE. Epidemiologic surveillance of cutaneous fungal infection in the United States from 1999 to 2002. J Am Acad Dermatol 2004;50:748-52.

2. Lipner SR, Scher RK. Onychomycosis: clinical overview and diagnosis. J Am Acad Dermatol 2019;80:835-51.

3. Lipner SR, Scher RK. Onychomycosis: treatment and prevention of recurrence. J Am Acad Dermatol 2019;80:853-67.

4. Gupta AK, Ahmad I, Borst I, Summerbell RC. Detection of xanthomegnin in epidermal materials infected with Trichophyton rubrum. J Invest Dermatol 2000;115:901-5.

5. Vural E, Winfield HL, Shingleton AW, Horn TD, Shafirstein G. The effects of laser irradiation on Trichophyton rubrum growth.
Lasers Med Sci 2008;23:349-53.

6. Gupta AK, Versteeg SG. A critical review of improvement rates for laser therapy used to treat toenail onychomycosis. J Eur Acad Dermatol Venereol 2017;31:1111-8.

7. Heinlin J, Maisch T, Zimmermann JL, Shimizu T, Holzmann T, Simon M, et al. Contact-free inactivation of Trichophyton rubrum and Microsporum canis by cold atmospheric plasma treatment. Future Microbiol 2013;8:1097-106.

8. Lipner SR, Friedman G, Scher RK. Pilot study to evaluate a plasma device for the treatment of onychomycosis. Clin Exp Dermatol 2017;42:295-8.

9. Moon IJ, Won $\mathrm{CH}$. Review of the current state of medical plasma technology and its potential applications. Med Lasers 2018;7:1-5

10. Cheng KY, Lin ZH, Cheng YP, Chiu HY, Yeh NL, Wu TK, et al. Wound healing in streptozotocin-induced diabetic rats using atmospheric-pressure argon plasma jet. Sci Rep 2018;8:12214.

11. Fitzpatrick R, Bernstein E, lyer S, Brown D, Andrews P, Penny K. A histopathologic evaluation of the Plasma Skin Regeneration System (PSR) versus a standard carbon dioxide resurfacing laser in an animal model. Lasers Surg Med 2008;40:93-9. 